\title{
Assessing portion size in ethnic minorities in the U.K.: A systematic review of existing instruments
}

\author{
E. Almiron-Roig ${ }^{1}$, A. Aitken ${ }^{2}$, Catherine Galloway ${ }^{1}$ and Basma Ellahi ${ }^{2}$ \\ ${ }^{1}$ MRC Human Nutrition Research, Cambridge, CB19NL, UK and ${ }^{2}$ Faculty of Health and Social Care, University of \\ Chester, CH1 4BJ, UK
}

Dietary assessment in ethnic minorities is critical for surveillance programmes and for implementing effective interventions in these groups. A major challenge is the accurate estimation of portion sizes for traditionally consumed foods and dishes.

To explore how to best apply standard portion size estimation techniques in ethnic minorities, we systematically reviewed the literature on portion size estimation elements (PSEE) applicable to UK ethnic minority groups. PSEE were defined as a component of the dietary assessment method designed to help quantify the amount of food reportedly consumed and including: portion size estimation aids (e,g. photographs, household utensils); guidance food amounts (e.g. 'large', 'medium', 'small' vs. a reference amount), natural units (e.g. 1 slice) and volume units (gr, oz, ml). We searched 24 electronic databases, $1 \mathrm{PhD}$ thesis and a review paper for records published between 1910-2014 using standard systematic review methods and complemented searches with cross-references.

A total of 4041 titles were identified from which 110 abstracts were screened and from which 36 eligible records were retained for full analysis. A total of 24 PSEE from 21 eligible sources (1984-2012) related to migrants were analysed. A sample of studies conducted in relevant original countries were also analysed but are not reported here. The most common PSEE ( $29 \%$ ) were combination tools, i.e. using more than one PSEE in the same dietary assessment instrument (e.g. guidance amounts, food models and household measures as part of an FFQ). Guidance amounts as part of food guide pyramids, sample menus and other guides accounted for $21 \%$ of all PSEE, followed by guidance amounts in FFQs/other questionnaires; photographs and drawings; and food models plus food scales (16.5\% each). Stand-alone photographs, food atlases, drawings and pictorial guides (2D tools), accounted for $47 \%$ of the noncombination PSEE, while food models and food scales $24 \%$. FFQs were the most common dietary assessment instrument where PSEEs were used $(42 \%)$. The predominant target population was the South Asian/Indian community (13 PSEE identified), followed by mixed populations (4), Chinese (3), African and other Black (3), Cuban/Puerto Rican (2), Afro-Caribbean, Arab and Asian (1 each). Tools most commonly used for the South Asian community included food scales, photographs and drawings; and for Chinese migrants food models and FFQ guidance amounts. Half of the PSEE $(54 \%)$ had been validated, and another $54 \%$ had been piloted or tested for reliability, typically as part of the full dietary assessment instrument, with the most common gold-standard being a $24 \mathrm{~h}$ recall $(36 \%)$. Of the 14 PSEE that had been validated $10(71 \%)$ had also been piloted or tested for reliability; $46 \%$ of PSEE used primary data to inform its design (e.g. interviews).

Several issues relevant to the development of ethnic-specific dietary assessment tools were identified including knowledge of customary portion sizes by sex and age; use of traditional household utensil measures; literacy levels of the target population; and combining multiple PSEE to increase accuracy.

1. Higgins JPT \& Green S (2011) Cochrane Handbook for Systematic Reviews of Interventions Version 5.1.0 [updated March 2011$]$ The Cochrane Collaboration. 